\title{
Construction of Cyclically Permutable Codes From Cyclic Codes
}

\author{
Ting-Ya Yang ${ }^{\mathrm{a}}$, Houshou Chen ${ }^{\mathrm{a}, ~}{ }^{\text {, }}$ Kuo-Cheng Chung ${ }^{\mathrm{a}}$ \\ aDept. of Electrical Engineering and Graduate Institute of Communication Engineering \\ National Chung Hsing University, 250, Kuo Kuang Rd., Taichung 402, Taiwan \\ *Corresponding Author: houshou@dragon.nchu.edu.tw
}

\begin{abstract}
Cyclically permutable codes (CPCs) are important to communication networks, e.g., multiple access collision channels without feedback and frequency-hopping spread spectrum communication channels. A CPC is a block code of length $\mathrm{n}$ such that each codeword has full cyclic order $\mathrm{n}$ and all codewords are cyclically distinct. This study investigates the characteristics of finite fields to develop an efficient algorithm to find a CPC from a $p$-ary cyclic code, where $\mathrm{p}$ is a prime number. In this paper, the Galois field Fourier transform technique is used to generate a CPC of either primitive or non-primitive length.
\end{abstract}

Keywords: Cyclic codes, finite field, cyclically permutable codes, BCH codes, Reed-Solomon codes.

\section{Introduction}

For the past few years, cyclically permutable codes and their applications in communication networks, e.g. multiple access collision channel without feedback (1) and frequencyhopping spread spectrum communications channels (2), (3), and digital watermarking (4), (5) have become increasingly important. A cyclic code (6) is defined as a linear block code such that any cyclic shift of every codeword yields another codeword. Gilbert (7) defined a cyclically permutable code (CPC) as a block code of length $n$, such that each codeword has cyclic order $\$ n \$$ and any cyclic shift of all codewords are distinct, i.e., no codeword in CPC can be obtained by any cyclic shift of another codeword. Maracle and Wolverton (8) proposed an algorithm for constructing the cyclically inequivalent subset. However, an existing $p$-ary cyclic codes usually do not enable this efficient construction.

A cyclically permutable code of length $n$ can be obtained directly from a cyclic code by partitioning this cyclic code into cyclically equivalent subsets, each consisting of all cyclic shift of a codewords, and then choosing any one codeword from those subsets of size $\$ \mathrm{n} \$$. However, it is required that the CPC must be effectively constructed from this cyclic code. N. Q. A. L. Gyorfi and J. L. Massey (9) proposed an encoding procedure for obtaining cyclically equivalent subsets and formed a CPC from a maximum-distance-separable (MDS) code, such as abReedSolomon (RS) code of length $p-1$ or a generalized Berlekamop-Justesen (BJ) code of length $p+1$, both with dimension $k$ and over $F$. More precisely, they constructed a CPC with $p^{k-1}$ codewords from a RS code or a CPC with $p^{k-2}$ codewords from a BJ code.

The design of a difference family and several constructions of constant-weight CPCs are presented in (10) where the authors proposed combinatorial constructions to constrcut a CPC that have other coding applications. In (11), the authors proposed the use of algebraic property of a binary cyclic code, such as the generator polynomial in timedomain, for an efficient and systematic construction of a CPC from this binary cyclic code.

In this paper, we use the Galois field Fourier transform method to form a CPC from a $p$-ary cyclic code of length $n$ where $n$ is a divisor of $p^{m-1}$. Let $\alpha$ be an element with multiplication order $n$. This study extends the results of (9) in twofold advantages. First, for a cyclic code of nonprimitive length $n=\left(p^{m-1}\right) / s, s>1$, and dimension $k$, we can construct a CPC with $s \cdot p^{k-m}$ codewords. The CPC constructed here has $s$ times more codewords compared to the CPC constructed in (9). Secondly, let $\alpha^{i}, i>1$, be a nonzero of a RS code of primitive length $p-1$ and assume $i$ and $p-1$ are relative prime, we can then construct a CPC which has more than $p^{k-1}$ codewords. The remainder of this paper is organized as follows. In Section, we describe the Galois Field Fourier transforms for a $p$-ary cyclic code. Section III proposes an efficient construction for a CPC from the cyclic codes and provides some CPC examples with 
constructive discussions. Finally, Section IV presents the conclusion.

\section{Galois Field Fourier Transform Methods}

For signal processing applications, there are several discrete Fourier transforms applied to complex fields (6). Fourier transforms also exists in the Galois field $G F(q)$, which is important in the study and processing of cyclic codes. As opposed to (11), which used the time-domain to find a CPC, this study proposes the use of the frequency domain as an efficient method to find many CPCs from cyclic codes. Cyclic codes are defined as codes whose codewords have certain specified spectral components that are equal to zeros. The most important cyclic codes studied in this paper are the Reed-Solomon (RS) codes and BossChaudhur-Hochquenghen $(\mathrm{BCH})$ codes, which are used to find CPCs. In this section, we describe the constructions of the Galois field Fourier transform (GFFT) to define cyclic codes, and we use the GFFT for cyclic codes to find the CPC. Galois field Fourier transform is a linear operator described by a matrix multiplication. Let $v=\left(v_{0}, v_{1}, \ldots, v_{n-1}\right)$ be a vector over $F_{q}{ }^{m}$ of length $n \mid q^{m}-1$ for some positive integer $m$. Let $\alpha \in G F\left(q^{m}\right)$ have order $n$. The GFFT of $v$ is the vector $V=\left(V_{0}, V_{1}, \ldots, V_{n-1}\right)$ defined as

$$
\left(\begin{array}{c}
V_{0} \\
V_{1} \\
\vdots \\
V_{n-1}
\end{array}\right)=\left(\alpha^{i j}\right) \cdot\left(\begin{array}{c}
v_{0} \\
v_{1} \\
\vdots \\
v_{n-1}
\end{array}\right)
$$

where $\left(\alpha^{i j}\right)$ is a matrix

$$
\left(\alpha^{i j}\right)=\left(\begin{array}{ccccc}
1 & 1 & 1 & \cdots & 1 \\
1 & \alpha^{1} & \alpha^{2} & \cdots & \alpha^{(n-1)} \\
1 & \alpha^{2} & \alpha^{4} & \cdots & \alpha^{2(n-1)} \\
\vdots & \vdots & \vdots & & \vdots \\
1 & \alpha^{(n-1)} & \alpha^{2(n-1)} & \cdots & \alpha^{(n-1)(n-1)}
\end{array}\right)
$$

The GFFT can be also written by the formula

$V_{j}=\sum_{i=0}^{n-1} \alpha^{i j} v_{i} \quad j=0,1, \cdots, n-1$.

We can use $v \leftrightarrow V$ to denote the Fourier transform relationship between $v$ and $V$. Similarly, the inverse GFFT of the vector $V=\left(V_{0}, V_{1}, \ldots, V_{n-1}\right)$ can be showns to be $\left(\begin{array}{c}v_{0} \\ v_{1} \\ \vdots \\ v_{n-1}\end{array}\right)=\frac{1}{n}\left(\alpha^{-i j}\right) \cdot\left(\begin{array}{c}V_{0} \\ V_{1} \\ \vdots \\ V_{n-1}\end{array}\right)$

where $\left(\alpha^{-i j}\right)$ is a matrix

$\left(\alpha^{-i j}\right)=\left(\begin{array}{ccccc}1 & 1 & 1 & \cdots & 1 \\ 1 & \alpha^{-1} & \alpha^{-2} & \cdots & \alpha^{-(n-1)} \\ 1 & \alpha^{-2} & \alpha^{-4} & \cdots & \alpha^{-2(n-1)} \\ \vdots & \vdots & \vdots & & \vdots \\ 1 & \alpha^{-(n-1)} & \alpha^{-2(n-1)} & \cdots & \alpha^{-(n-1)(n-1)}\end{array}\right)$

The inverse GFFT can also be written as

$v_{i}=n^{-1} \sum_{j=0}^{n-1} \alpha^{-i j} V_{j}$

where $n^{-1}$ is the vector multiplicative inverse of $n$. The spectrum of the polynomial $v(x)=v_{0}+v_{1} x+\ldots+v_{n-1} x^{n-1}$ is the GFFT of $v=\left(v_{0}, v_{1}, \ldots, v_{n-1}\right)$, and the vector $v$ is in the time domain, and its corresponding transform $V$ is in the frequency domain. Given a polynomial $v(x)$, we can show that

$V_{j}=\sum_{i=0}^{n-1} v_{i}\left(\alpha^{j}\right)^{i}=v\left(\alpha^{j}\right)$

The $j$-th component of the GFFT of $v$ is obtained by evaluating $v(x)$ at $x=\alpha^{j}$. Similarly, we can write the $i$-th component of $v$ as

$v_{i}=\frac{1}{n} \sum_{j=0}^{n-1} V_{j}\left(\alpha^{-i}\right)^{j}=\frac{1}{n} V\left(\alpha^{-i}\right)$

Then, $\alpha^{-i}$ is a zero of $V(x)$ if and only if the $i$-th frequency component $v_{i}$ of the inverse transform $v$ of $V$ equals zero. When the zeros of a cyclic code are $\left\{\alpha^{1}, \alpha^{2}, \ldots, \alpha^{n-k}\right\}$, then the inverse of nonzeros are

$\left\{\alpha^{-(n-k+1)}, \alpha^{-(n-k+2)}, \cdots, \alpha^{-(n-1)}\right\}=\left\{\alpha^{k-1}, \alpha^{k-2}, \cdots, \alpha^{1}\right\}$

We can then use the zeors and the inverse of the nonzeros of a cyclic code to form the parity check matrix $H$ and the generator matrix $G$ as

$H=\left(\begin{array}{c}\left(\alpha^{1}\right)^{j} \\ \left(\alpha^{2}\right)^{j} \\ \vdots \\ \left(\alpha^{n-k}\right)^{j}\end{array}\right) \cdot G=\left(\begin{array}{c}\left(\alpha^{0}\right)^{j} \\ \left(\alpha^{1}\right)^{j} \\ \vdots \\ \left(\alpha^{n-1}\right)^{j}\end{array}\right)$

where $j=0,1, \ldots, n-1$. 


\section{Construction of Cyclically Permutable Codes}

CPCs are based on the characteristics of cyclic codes, where the cyclically shifted codewords occupy the same subspace, with each CPC codeword belonging to each subspace. Gillbert (3) defined a CPC as a binary block code, the codewords of which are cyclically distinct and have full order. This study proposes efficient methods that can be used to find the CPC, and propose the construction of CPCs using $p$-ary linear cyclic $(n, k, d)$ codes, where $n, k$ and $d$ are the block length, dimension and minimum Hamming distance, respectively, and those codes with code digits in $G F(q)$ and $p$ can be a prime number. Moreover, this paper presents a cyclic code that can be used to find more CPCs compared with (6), and $n$ can have both primitive and non-primitive lengths.

\section{1 $\alpha^{-i}$ is a $(n, k)$ cyclic code zero root, then $\operatorname{gcd}(n, i)=1$}

Lemma: A Consider the binary $(n, k, d) \mathrm{BCH}$ code, where $n$ is prime and has primitive length, and where $n=2^{m}$ 1 , and has $2^{k}-2$ codewords with full order; then, we can obtain a CPC using $\left(2^{k}-2\right) / n$.

Proof: Let $\left\{\alpha^{s_{1}}, \alpha^{s_{2}}, \cdots, \alpha^{s_{l}}\right\}$ be a zero root and all its cyclotomic cosets, and the non-zero inverse be $\left\{\alpha^{0}, \cdots, \alpha^{s_{l}}\right\} /\{$ zeros $\}=\left\{\right.$ nonzeros $\left.^{-1}\right\}=\left\{\alpha^{t_{0}}, \alpha^{t_{1}}, \cdots, \alpha^{t_{r}}\right\} \quad$, where $\alpha^{0}=\alpha^{t_{0}}$. Let $k=1+r \cdot m$ be the binary $(n, k, d) \mathrm{BCH}$ code, where the codewords have $2^{k}=2 \cdot 2^{m \cdot r}$. Substituting a nonzero inverse into (3) to generate the matrix, we have

$G=\left(\begin{array}{c}\alpha^{0} \\ \alpha^{t_{1} \cdot 1} \\ \vdots \\ \alpha^{t_{1} \cdot m} \\ \alpha^{t_{2} \cdot 1} \\ \vdots \\ \alpha^{t_{2} \cdot m} \\ \alpha^{t_{r} \cdot 1} \\ \vdots \\ \alpha^{t_{r} \cdot m}\end{array}\right) \Rightarrow c=\left(u_{0}, u_{t_{1}}, u_{t_{2}}, \ldots, u_{t_{r}}\right) \cdot G$

where $u_{0} \in F_{2}$ and $u_{x} \in F_{2}{ }^{m}$, and $x=1, \ldots, r$; then, we can find the CPC. First, let $u_{t l}=1$ belong to $F_{2}{ }^{m}$ arbitrary value. We have a $2^{k-m}\left(2^{m}-1\right)=\left(2^{m}\right)^{r-1} \cdot 2 \cdot\left(2^{m}-1\right)$ codeword with full order, and the CPC number is $\left(2^{m}\right)^{r-1} \cdot 2$. The next set of $u_{t t}=0$ and $u_{t 2}=1$ and another still belongs to the arbitrary value $F_{2}{ }^{m}$. Similarly, we can calculate $\left(2^{m}\right)^{r-2} \cdot 2 \cdot\left(2^{m}-1\right)$ full order codewords, and these CPC numbers are $\left(2^{m}\right)^{r-2} \cdot 2$. If the CPC of the sequence obtained from $u_{t \prime}, \ldots, u_{t r}$ are zeros and $u_{t r}=1$, then the CPC number is $\left(2^{m}\right)^{r-r} \cdot 2=2$. Finally, there are total CPC induction formula components of $2\left[\left(2^{m}\right)^{r-1}+\left(2^{m}\right)^{r-}\right.$ $\left.{ }^{2}+\left(2^{m}\right)^{r-3}+\ldots+\left(2^{m}\right)^{1}+\left(2^{m}\right)^{0}\right]=2 \cdot\left(2^{m r}-1\right) /\left(2^{m}-1\right)=\left(2^{m} \cdot r+1\right.$ $2) /\left(2^{m}-1\right)=\left(2^{k}-2\right) / n$. We obtained the desired result when $n$ is prime and has primitive length, and where the total CPC number is $\left(2^{k}-2\right) / n$.

EXAMPLE 1: Consider a $(31,16,7) \mathrm{BCH}$ code over $F_{2}$. We can extend it to $F_{2}^{5}=\left(F_{2}[x]\right) /\left(x^{5}+x^{2}+1\right)$, where the degree is $m=5$ and where $n=2^{5}-1$ is primitive and has primitive length. The cyclotomic coset is

$\{1,2,4,8,16\}$

$\{3,6,12,24,17\}$

$\{5,10,20,9,18\}$

$\{7,14,28,25,19\}$

$\{11,22,13,26,21\}$

$\{15,30,29,27,13\}$

Let the zeros be $\left\{\alpha^{1}, \alpha^{3}, \alpha^{5}\right\}$, and another element $\left\{\alpha^{7}, \alpha^{11}, \alpha^{15}\right\}$ be non-zero. Then, the non-zero inverse is $\left\{\alpha^{-7}, \alpha^{-11}, \alpha^{-15}\right\}=\left\{\alpha^{1}, \alpha^{3}, \alpha^{5}\right\}$. Furthermore, we can obtain the generated matrix

$G=\left(\begin{array}{c}\alpha^{0} \\ \alpha^{1} \\ \vdots \\ \alpha^{3} \\ \vdots \\ \alpha^{5} \\ \vdots\end{array}\right) \Rightarrow c=\left(u_{0}, u_{1} \cdots, u_{3} \cdots, u_{5} \cdots\right) \cdot G$

where $u_{0} \in F_{2}$ and $u_{1}, u_{3}, u_{5} \in F_{2}{ }^{5}$. First, let $u_{1}=1$ and $u_{3}, u_{5} \in$ $F_{2}{ }^{5}$, be another element, then the CPC number is $2^{k-m}=2^{16-}$ ${ }^{5}=2^{11}$. Second, let $u_{1}=0, u_{3}=1$ and $u_{5}$ belong to $F_{2}{ }^{m}$, which is another element. The CPC number is $2^{k-2 m}=2^{16-10}=2^{6}$. Then, let $u_{1}=0, u_{3}=0$ and $u_{5}=1$, and the number of $2^{k-3 m}=2^{16-15}=2^{1}$ with CPCs. We obtain a total CPC number of $\left(2^{k}-2\right) / n=\left(2^{16}\right.$ $2) / 31$. Similarly, the same characteristics of in $p$-ary exist.

\section{$3.2(n, k)$ cyclic codes with non-primitive length $s \neq 1$}

We use GFFT for RS codes defined as

$\left(c_{0}, \cdots, c_{n-1}\right)=\left(u_{0}, \cdots, u_{k-1}\right) \cdot\left(\begin{array}{c}\left(\alpha^{0}\right)^{i} \\ \vdots \\ \left(\alpha^{k-1}\right)^{i}\end{array}\right)$

We consider the codes with zeros $\left\{\alpha^{1}, \alpha^{2}, \cdots, \alpha^{n-k}\right\}$, then, we evaluate $\quad v\left(\alpha^{1}\right)=v\left(\alpha^{2}\right)=\cdots=v\left(\alpha^{n-k}\right)$ and $V_{1}=V_{2}=\ldots=V_{n-k}=0$.

We use the definition in (2) as 


$$
\begin{aligned}
& \left(\begin{array}{c}
c_{0} \\
\vdots \\
c_{n-1}
\end{array}\right)=\left(\begin{array}{c}
v_{0} \\
\vdots \\
v_{n-1}
\end{array}\right)=\frac{1}{n} \cdot\left(\alpha^{-i j}\right) \cdot\left(\begin{array}{c}
V_{0} \\
V_{1} \\
\vdots \\
V_{n-1}
\end{array}\right) \\
& \Rightarrow\left(\begin{array}{c}
c_{0} \\
\vdots \\
c_{n-1}
\end{array}\right)=\left(\begin{array}{llll}
\frac{1}{n} v_{0} & \frac{1}{n} v_{n-k+1} & \cdots & \frac{1}{n} v_{n-1}
\end{array}\right) \cdot\left(\alpha^{-i j}\right)
\end{aligned}
$$

for $1 \leq i \leq n-k$ and $j=0, n-k+1, \ldots, n-1$. Let $u_{0}=(1 / n) \cdot V_{0}$, $u_{1}=(1 / n) \cdot V_{n-1}, u_{2}=(1 / n) \cdot V_{n-2}, \ldots, u_{k-1}=(1 / n) \cdot V_{n-k+1}$ then we can obtain

$u_{j}=\frac{1}{n} V_{n-j}=\frac{1}{n} V_{j}=\frac{1}{n} v\left(\alpha^{-j}\right)=\frac{1}{n} c\left(\alpha^{-j}\right)$

Consider $c^{(t)}(x)$ as the $t$ right shift of $c(x)$ component of

$u_{j}^{(t)}=\frac{1}{n} c^{(t)}\left(\alpha^{-j}\right)=\frac{1}{n} \alpha^{-j t} \cdot c\left(\alpha^{-j}\right)=\alpha^{-j t} \cdot \frac{1}{n} c\left(\alpha^{-j}\right)$

Finally, we have

$u_{j}^{(t)}=\alpha^{-j t} \cdot u_{j}$

Consider $(p+1, k, p+2-k) \mathrm{RS}$ code over $F_{p}$, where $n=p+1$ is of non-primitive length. Let $n=p+1$ and $s=\left(p^{2}-1\right) /(p+1)=p$ 1 ; then, $o(\beta)=p^{2}-1$ and $o(\alpha)=p+1$, and we can obtain $\alpha=\beta^{\frac{p^{2}-1}{p+1}}=\beta^{p-1}=\beta^{s}$. Using (3), we obtain $u_{1}{ }^{(t)}=\left(\alpha^{-t}\right) u_{1} \Rightarrow$ $\left(\beta^{-(p-1) \cdot t}\right) u_{1} \Rightarrow\left(\beta^{-s \cdot t}\right)$.

$u_{1}=\beta^{0} \quad \Rightarrow \beta^{-s}, \beta^{-2 s}, \cdots, \beta^{-(n-1) s}$

$u_{1}=\beta^{-1} \Rightarrow \beta^{-(s+1)}, \beta^{-(2 s+1)}, \cdots, \beta^{-((n-1) s+1)}$

$u_{1}=\beta^{-2} \Rightarrow \beta^{-(s+2)}, \beta^{-(2 s+2)}, \cdots, \beta^{-((n-1) s+2)}$

$u_{1}=\beta^{-3} \Rightarrow \beta^{-(s+3)}, \beta^{-(2 s+3)}, \cdots, \beta^{-((n-1) s+3)}$

$u_{1}=\beta^{-(s-1)} \Rightarrow \beta^{-(2 s-1)}, \beta^{-(3 s-1)}, \cdots, \beta^{-(n s-1)}$

In this study, we generate $\left[\left(p^{2}-1\right) /(p+1)\right] \cdot p^{k-2}=s \cdot p^{k-2}$. Comparing CPCs with [6] only have $p^{k-2}$ CPCs, and we obtain additional CPCs. Further, in [6], the code length is only primitive length.

EXAMPLE 2: For $[35,25,4] \mathrm{BCH}$ codes over $F_{2}{ }^{12}$, the cyclotomic coset is

$\{3,6,12,24,13,26,17,34,33,31,27,9\}$

First, we define $s=\left(2^{m}-1\right) / n=\left(2^{12}-1\right) / 35=4095 / 35=117$, $\alpha=\beta^{s}=\beta^{117}$ and $o(\alpha)=35$ and $o(\beta)=2^{12}-1$. Consider the zeros as $\left\{\alpha^{5}, \alpha^{7}, \alpha^{15}\right\}$, another is non-zero, where the nonzero inverse is $\left\{\alpha^{0}, \alpha^{1}, \alpha^{3}\right\}$. The generated matrix component of
$G=\left(\begin{array}{c}\left(\alpha^{0}\right)^{i} \\ \vdots \\ \left(\alpha^{1}\right)^{i} \\ \vdots \\ \left(\alpha^{3}\right)^{i} \\ \vdots\end{array}\right) \Rightarrow c=\left(u_{0}, \quad u_{1}, \quad u_{3}\right) \cdot G$

where $u_{0} \in F_{2}$ and $u_{1}, u_{3} \in F_{2}{ }^{12}$. First, let $u_{1}=1$ and $u_{3} \in F_{2}{ }^{12}$ be another element in the codeword $s \cdot 2^{k-m} \cdot\left(2^{12}-1\right)=117 \cdot 2^{13}$ - $\left(2^{12}-1\right)$ with full order.

Then, the CPC number is $s \cdot 2^{k-m}=117 \cdot 2^{25-12}=117 \cdot 2^{13}$. Second, set $u_{1}=0$ and $u_{3}=1$ in the codeword $s \cdot 2^{k-2 m}\left(2^{12}-1\right)=s$ $\cdot 2^{25-24} \cdot\left(2^{12}-1\right)=117 \cdot 2 \cdot\left(2^{12}-1\right)$ with full order, then the CPC number is $s \cdot 2^{k-2 m}=s \cdot 2^{25-24}=117 \cdot 2$. Then, we obtain the total CPC number of $s \cdot 2^{13}+s \cdot 2^{1}=117 \cdot\left(2^{13}+2^{1}\right)$.

EXAMPLE 3: Consider a $(6, k, d=n-k)$, which is RS code over $F_{5}$ and $m=2$, the code is non-prime and has primitive length. We can calculate $s=\left(p^{2}-1\right) /(p+1)=\left(5^{2}-1\right) / 6=p-=14$ and $o(\alpha)=6, o(\beta)=p^{2}-1=5^{2}-1=24$. Next, we consider a $(6$, $3,4) \mathrm{RS}$ code with $\left\{\alpha^{3}, \alpha^{2}, \alpha^{4}\right\}$ as zeros and $\left\{\alpha^{0}, \alpha^{1}, \alpha^{5}\right\}$ as non-zeros. The generated matrix component of $G=\left(\begin{array}{l}\left(\alpha^{0}\right)^{i} \\ \left.\alpha^{1}\right)^{i} \\ \left(\alpha^{5}\right)^{i}\end{array}\right) \Rightarrow c=\left(u_{0}, \quad u_{1}, \quad u_{5}\right) \cdot G$

where $u_{0} \in F_{5}$ and $u_{1}, u_{5} \in F_{5}^{2}$. When $u_{1}=1, u_{5} \in F_{5}^{2}$ the CPC number is $(p-1) \cdot p^{k-1}=s \cdot p^{k-1}=4 \cdot 5^{3-1}$. Also, when $u_{1}=0, u_{5}=1$, the CPC number is $(p-1) \cdot\left(p^{k-2}\right)=s \cdot p^{k-2}=4 \cdot 5^{3-2}=20$. Finally, the total CPC number is $(p-1) \cdot\left(p^{k-1}+p^{k-2}\right)=4 \cdot\left(5^{3-1}+5^{3-2}\right)$.

We can obtain $u_{1}{ }^{(t)}=\left(\alpha^{-t}\right) \cdot u_{1} \Rightarrow\left(\beta^{-4 \cdot t}\right) \cdot u_{1}=\beta^{-4 \cdot t}$

$$
\begin{aligned}
& u_{1}=\beta^{0} \quad \Rightarrow \beta^{0}, \beta^{-4}, \beta^{-8}, \beta^{-12}, \beta^{-16}, \beta^{-20} \\
& u_{1}=\beta^{-1} \quad \Rightarrow \beta^{-1}, \beta^{-5}, \beta^{-9}, \beta^{-13}, \beta^{-17}, \beta^{-21} \\
& u_{1}=\beta^{-2} \quad \Rightarrow \beta^{-2}, \beta^{-6}, \beta^{-10}, \beta^{-14}, \beta^{-18}, \beta^{-22} \\
& u_{1}=\beta^{-3} \quad \Rightarrow \beta^{-3}, \beta^{-7}, \beta^{-11}, \beta^{-15}, \beta^{-19}, \beta^{-23}
\end{aligned}
$$

\section{Conclusion and Discussion}

Cyclic codes are block codes in which a cyclic shift codeword generates another codeword belonging to the same subspace. With cyclically permutable codes (CPCs), the codewords are cyclically distinct and have full cyclic order. Although it is important to effectively determine CPCs from cyclic codes, no approach has thus far been proposed. In this paper, we study the characteristics of finite fields and cyclic codes, and propose an efficient CPC construction procedure. The construction method proposed here is more efficient than the RS-based construction proposed in (5) and (6). In the first case, when $\operatorname{gcd}(n, i)=1$, we can obtain more CPCs. 
Second, in (6), there is only the case where the CPC number comprises $s=1$ multiples, and we can obtain more than $s \geq 1$ multiples of CPC. Moreover, as opposed to (5) which used the time domain, we proposed to use the frequency domain as an efficient method to find the CPC from cyclic code. We have shown the construction of CPCs based on the binary mapping of some $p$-ary linear cyclic codes, and we noted that the code can have primitive and non-primitive length.

\section{References}

(1) L. Gyorfi, I. Vajda. "Constructions of protocol sequence for multiple access collision channel without feedback," IEEE Trans. Inform, Theory, pp. 17621765, 1993.

(2) W. Lam and D. V. Sarwate. "Time-hopping and frequency-hopping multiple-access packet communications," IEEE Tans, on Commun., pp. 875$888,1990$.

(3) R. Gagliardi, J. Robbins and H. Taylor, "Acquisition sequence in PPM communications," IEEE Trans. Inform, Theory, pp. 738-744, 1977.

(4) H. Inaba and H. Nakahara, "Notes on rotation-resistant digital watermark using radon transform," in Proc. Int. Symp. Information Theory and Its Applications (ISITA2004), Parma, Italy, pp. 310-315, Oct. 2004.

(5) I. Cox, M. Miller, J. Bloom, J. Fridrich, and T. Kalker, Digital Watermarking and Steganography, 2nd ed. San Mateo, CA: Morgan Kaufmann, 2007.

(6) T. K. Moon, Error Correction Coding: Mathematical Methods And Algorithms. Wiley Inter-science, pp. 269276, 2005.

(7) E. N. Gillbert, "Cyclically permutable error-correcting codes," IEEE Trans. Inform, Theory, vol. 9, pp. 175-182, Jul. 1963.

(8) D. E. Maracle and C. T. Wolverton, "Generating cyclically permutable codes," IEEE Trans. Inform, Theory, vol. IT-20, no. 4, pp. 554-555, Jul. 1974.

(9) N. Q. A. L. Gyorfi and J. L. Massey, "Constructions of binary constant weight cyclic codes and cyclically permutable codes," IEEE Trans. Inform, Theory, vol. 38, no. 3, pp. 940-949, May 1992.

(10) S. Bitan and T. Etzion, "Constructions for optimal constant weight cyclically permutable codes and difference families," IEEE Trans. Inform, Theory, vol. 41, no. 1, pp. 77-87, Jan. 1995.

(11) Kuribayashi and H. Tanaka, "How to Generate Cyclically Permutable Codes from Cyclic Codes," IEEE
Trans. Inform, Theory, vol. 52, no. 10, pp. 4660-4663, Oct. 2006 